\title{
THE NUMBER OF EDGES ON GENERALIZATIONS OF PALEY GRAPHS
}

\author{
LAWRENCE SZE
}

(Received 21 August 1997)

\begin{abstract}
Evans, Pulham, and Sheenan computed the number of complete 4-subgraphs of Paley graphs by counting the number of edges of the subgraph containing only those nodes $x$ for which $x$ and $x-1$ are quadratic residues. Here we obtain formulae for the number of edges of generalizations of these subgraphs using Gaussian hypergeometric series and elliptic curves. Such formulae are simple in several infinite families, including those studied by Evans, Pulham, and Sheenan.
\end{abstract}

2000 Mathematics Subject Classification. 05C30, 11L99.

1. Preliminaries. Let $G(p)$ be a graph with $p$ vertices, and let $G(p)^{c}$ denote its complement, the graph where $x y$ is an edge if and only if $x y$ is not an edge of $G(p)$. If $p \equiv 1(\bmod 4)$ is prime, then let $P(p)$ denote the Paley graph whose vertices are in $G F(p)$, the finite field with $p$ elements. This is the graph where $x y$ is an edge if and only if $x-y$ is a quadratic residue modulo $p$. Motivated by a conjecture of Erdös, which turned out to be false, Evans, Pulham, and Sheenan [2] computed $k_{4}(P(p))+$ $k_{4}\left(P(p)^{c}\right)$, where $k_{m}(G)$ denotes the number of complete $m$-subgraphs of G. Counting the number of edges of $G^{*}(p)$, the subgraph of $P(p)$ containing only those nodes $x$ for which $x$ and $x-1$ are both quadratic residues, was the main obstacle in obtaining their result. They showed [2, Proposition 4] that if $p=4 y^{2}+x^{2}$, then the number of edges in $G^{*}(p)$ is

$$
\frac{p^{2}-22 p+4 x^{2}+81}{64} .
$$

We compute the number of edges of $G(i, t, p)$, natural generalizations of $G^{*}(p)$.

Throughout $p$ is an odd prime, and $G F(p)$ is the finite field with $p$ elements. Furthermore let $G F(p)^{\times}$denote the nonzero elements of $G F(p)$, and let $G F(p)^{\times 2}$ denote the nonzero squares. For convenience we let $\phi(\cdot)$ denote the Legendre symbol $(\cdot / p)$ extended to $G F(p)$ under the convention that $\phi(0):=0$. If $n$ is an integer, then $\operatorname{ord}_{p}(n)$ is the power of $p$ dividing $n$ and $\operatorname{ord}_{p}(a / b):=\operatorname{ord}_{p}(a)-\operatorname{ord}_{p}(b)$.

DEFINITION 1.1. Let $1 \leq i \leq 8$ be an integer. If $p$ is an odd prime and $t$ a nonzero integer, then the generalized Paley graph $G(i, t, p)$ is the directed graph whose edge set $E(i, t, p)$ is

$$
\begin{gathered}
E(i, t, p):=\left\{x \rightarrow y \mid(x, y) \in G F(p)^{\times^{2}} \times G F(p)^{\times^{2}}, \phi(1-x)=(-1)^{\lfloor(i-1) / 4\rfloor},\right. \\
\\
\left.\phi(1-y)=(-1)^{\lfloor(i-1) / 2\rfloor}, \phi(x-t y)=(-1)^{i-1}\right\},
\end{gathered}
$$

where $\lfloor\cdot\rfloor$ denotes the greatest integer function. 
The reader should note that edges can be loops, and also that the index $i$ simply keeps track of the eight nontrivial combinations of signs for $\phi(1-x), \phi(1-y)$, and $\phi(x-t y)$. If $p \equiv 1(\bmod 4)$, then since $\phi(x-y)=\phi(y-x)$ the graph $G(1,1, p)$ is a double cover of $G^{*}(p)$.

We recall the definition of a Gaussian hypergeometric series as defined in [3]. Extend all characters $\chi$ of $G F(p)^{\times}$to $G F(p)$ by setting $\chi(0):=0$. If $A$ and $B$ are two characters of $G F(p)$, then $\left(\begin{array}{l}A \\ B\end{array}\right)$ is defined by the normalized Jacobi sum

$$
\left(\begin{array}{l}
A \\
B
\end{array}\right):=\frac{B(-1)}{p} J(A, \bar{B})=\frac{B(-1)}{p} \sum_{x \in G F(p)} A(x) \bar{B}(1-x) .
$$

DEFINITION 1.2. Given characters $A_{0}, A_{1}, \ldots A_{n}$, and $B_{1}, B 2, \ldots B_{n}$ of $G F(p)$, let

$$
{ }_{n+1} F_{n}\left(\begin{array}{ccc}
A_{0}, & A_{1}, & \ldots A_{n} \\
& B_{1}, & \ldots B_{n}
\end{array}\right)
$$

be the Gaussian hypergeometric series defined by

$$
{ }_{n+1} F_{n}\left(\begin{array}{ccc}
A_{0}, & A_{1}, & \ldots A_{n} \\
& B_{1}, & \ldots B_{n}
\end{array}\right):=\frac{p}{p-1} \sum_{\chi}\left(\begin{array}{c}
A_{o} \chi \\
\chi
\end{array}\right)\left(\begin{array}{c}
A_{1} \chi \\
B_{1} \chi
\end{array}\right) \ldots\left(\begin{array}{c}
A_{n} \chi \\
B_{n} \chi
\end{array}\right) \chi(t),
$$

where the summation is over all the characters $\chi$ of $G F(p)$.

Although the Gaussian hypergeometric series depend on the prime $p$, we suppress its dependence in the notation under the assumption that the prime will be clear from context.

For our purposes it will be important to evaluate

$$
{ }_{2} F_{1}(t):={ }_{2} F_{1}\left(\begin{array}{cc}
\phi & \phi \\
& \varepsilon
\end{array} t\right), \quad{ }_{3} F_{2}(t):={ }_{3} F_{2}\left(\begin{array}{ccc}
\phi & \phi & \phi \\
& \varepsilon & \varepsilon
\end{array}\right),
$$

where $\varepsilon$ is the identity (i.e., $\varepsilon(x)=1$ for $x \neq 0$ ). In [3] it was shown that

$$
\begin{gathered}
{ }_{2} F_{1}(t)=\frac{\phi(-1)}{p} \sum_{x \in G F(p)} \phi(x) \phi(1-x) \phi(1-t x), \\
{ }_{3} F_{2}(t)=\frac{\phi(-1)}{p^{2}} \sum_{x, y \in G F(p)} \phi(x) \phi(y) \phi(1-x) \phi(1-y) \phi(x-t y) .
\end{gathered}
$$

A useful alternative for computing these two Gaussian hypergeometric series was given in [4], where it was shown that they may also be expressed in terms of the number of points on special elliptic curves over $G F(p)$. Define elliptic curves ${ }_{2} E_{1}(t)$ and ${ }_{3} E_{2}(t)$ by

$$
\begin{gathered}
{ }_{2} E_{1}(t): y^{2}=x(x-1)(x-t), \\
{ }_{3} E_{2}(t): y^{2}=x^{3}-t^{2} x^{2}+\left(4 t^{3}-t^{4}\right) x+t^{6}-4 t^{5} .
\end{gathered}
$$

Denote the number of points on ${ }_{2} E_{1}(t)$ and ${ }_{3} E_{2}(t)$ over $G F(p)$ by

$$
\begin{gathered}
{ }_{2} N_{1}(t, p):=\left|\left\{(x, y) \in G F(p) \times G F(p) \mid y^{2}=x(x-1)(x-t)\right\}\right|, \\
{ }_{3} N_{2}(t, p):=\left|\left\{(x, y) \in G F(p) \times G F(p) \mid y^{2}=x^{3}-t^{2} x^{2}+\left(4 t^{3}-t^{4}\right) x+t^{6}-4 t^{5}\right\}\right| .
\end{gathered}
$$


Now define the Frobenius traces, ${ }_{2} a_{1}(t, p)$ and ${ }_{3} a_{2}(t, p)$ by

$$
\begin{aligned}
& { }_{2} a_{1}(t, p):=p-{ }_{2} N_{1}(t, p), \\
& { }_{3} a_{2}(t, p):=p-{ }_{3} N_{2}(t, p) .
\end{aligned}
$$

In this notation, the following two theorems were proved in [4].

THEOREM 1.3. If $t \in \mathbb{Q}-\{0,1\}$ and $p$ is an odd prime for which $\operatorname{ord}_{p}(t(t-1))=0$, then

$$
{ }_{2} F_{1}(t)=-\frac{\phi(-1){ }_{2} a_{1}(t, p)}{p} .
$$

THEOREM 1.4. If $\delta \in \mathbb{Q}-\{0,4\}$ and $p$ is an odd prime for which $\operatorname{ord}_{p}(\delta(\delta-4))=0$, then

$$
{ }_{3} F_{2}\left(\frac{4}{4-\delta}\right)=\frac{\phi\left(\delta^{2}-4 \delta\right)\left({ }_{3} a_{2}(\delta, p)^{2}-p\right)}{p^{2}} .
$$

2. Main theorems. Here we compute the number of edges of the graphs $G(i, t, p)$ when $t \not \equiv 0(\bmod P)$. Without loss of generality, we assume that $1 \leq t \leq p-1$, although for aesthetic reasons we write $t=-1$ rather than $t=p-1$.

LEMMA 2.1. If $p$ is an odd prime and $t \equiv \equiv(\bmod p)$, then

(i) $\sum_{x \in G F(p)} \phi\left(x^{2}-t\right)=-1$,

(ii) $\left|\left\{x \in G F(p) \mid \phi\left(x^{2}-t\right)=1\right\}\right|=(p-2-\phi(t)) / 2$,

(iii) $\left|\left\{x \in G F(p) \mid \phi\left(t-x^{2}\right)=1\right\}\right|=(p-1-\phi(t)-\phi(-1)) / 2$,

(iv) $\left|\left\{x \in G F(p) \mid \phi\left(1-x^{2} / t\right)=1\right\}\right|=(p-1-\phi(t)-\phi(-t)) / 2$.

Proof. (i) By Euler's criterion that $\phi(x) \equiv x^{(p-1) / 2}(\bmod p)$ for all $x \in G F(p)$, and the Binomial theorem we obtain

$$
\begin{aligned}
\sum_{x \in G F(p)} \phi\left(x^{2}-t\right) & \equiv \sum_{x \in G F(p)}\left(x^{2}-t\right)^{(p-1) / 2}(\bmod p) \\
& \equiv \sum_{x \in G F(p)} \sum_{r=0}^{(p-1) / 2}\left(\begin{array}{c}
(p-1) / 2 \\
r
\end{array}\right) x^{2} r(-1)^{(p-1) / 2-r} \quad(\bmod p) .
\end{aligned}
$$

Since $\sum_{x \in G F(p)}^{x^{k}} \equiv 0(\bmod p)$ for $0<k<p-1$, the above sum is

$$
\equiv \sum_{x \in G F(p)}(-t)^{(p-1) / 2}+x^{p-1} \equiv-1 \quad(\bmod p)
$$

Furthermore, this sum is odd because $\sum_{x \in G F(p)} \phi\left(x^{2}-t\right)=\phi(-t)+2 \sum_{x=1}^{(p-1) / 2} \phi\left(x^{2}-t\right)$. Therefore since $\left|\sum_{x \in G F(p)} \phi\left(x^{2}-t\right)\right|<p$, one easily concludes that the sum is -1 .

(ii) Define $S_{+}$and $S_{-}$by

$$
S_{ \pm}:=\left|\left\{x \in G F(p) \mid \phi\left(x^{2}-t\right)= \pm 1\right\}\right| .
$$

By (i), $S_{+}-S_{-}=-1$. This and the equation $S_{+}+S_{-}=p-(1+\phi(t))$ determine $S_{+}$.

(iii) and (iv) are also easy exercises. 
LemmA 2.2. Suppose $p$ is an odd prime and $t \equiv \equiv, 1(\bmod p)$. If $\gamma_{ \pm, \pm}(t, p)$ is defined by

$$
\gamma_{ \pm \pm}(t, p):=\left|\left\{x \in G F(p)^{\times} \mid \phi\left(1-x^{2}\right)= \pm 1, \phi\left(1-\frac{x^{2}}{t}\right)= \pm 1\right\}\right|,
$$

then

$$
\begin{aligned}
& \gamma_{++}(t, p)=\frac{1}{4}\left(p+\phi(-t) p_{2} F_{1}(t)-\phi(-1)-2 \phi(t)\right. \\
&\left.-\phi(-t)-2 \phi\left(t^{2}-t\right)-\phi(1-t)-\phi\left(t-t^{2}\right)-7\right), \\
& \gamma_{+-}(t, p)=\frac{1}{4}\left(p-\phi(-t) p_{2} F_{1}(t)-\phi(-1)-\phi(1-t)\right. \\
&\left.-\phi\left(t-t^{2}\right)+\phi(-t)+2 \phi\left(t^{2}-t\right)-3\right) \\
& \gamma_{-+}(t, p)=\frac{1}{4}\left(p-\phi(-t) p_{2} F_{1}(t)+\phi(-1)+\phi(1-t)\right. \\
&\left.\quad+\phi\left(t-t^{2}\right)-\phi(-t)-2 \phi\left(t^{2}-t\right)-3\right) \\
& \gamma_{--}(t, p)=\frac{1}{4}\left(p+\phi(-t) p_{2} F_{1}(t)+\phi(-1)+\phi(1-t)\right. \\
&\left.\quad+\phi\left(t-t^{2}\right)+\phi(-t)+2 \phi\left(t^{2}-t\right)-2 \phi(t)-3\right) .
\end{aligned}
$$

Proof. These formulae follow from four key relations. Since $t \not \equiv 0,1(\bmod p)$, it is clear that

$$
\begin{aligned}
\gamma_{++}(t, p)+\gamma_{--}( & t p)+\gamma_{+-}(t, p)+\gamma_{-+}(t, p) \\
& =\left|\left\{x \in G F(p)^{\times} \mid \phi\left(1-x^{2}\right) \neq 0, \phi\left(1-\frac{x^{2}}{t}\right) \neq 0\right\}\right| \\
& =p-4-\phi(t) .
\end{aligned}
$$

Similarly it is easy to see that

$$
\begin{aligned}
\gamma_{++}(t, p)+\gamma_{--}(t, p)-\gamma_{+-}( & t, p)-\gamma_{-+}(t, p) \\
= & \sum_{x \in G F(p)^{\times}} \phi\left(1-x^{2}\right) \phi\left(1-\frac{x^{2}}{t}\right) \\
& =-1+\sum_{x \in G F(p)} \phi\left(1-x^{2}\right) \phi\left(1-\frac{x^{2}}{t}\right) .
\end{aligned}
$$

Since $x^{2}$ represents each nonzero quadratic residue twice, replacing $x^{2}$ by $x$ in the above expression and then multiplying the summand by the weight $(1+\phi(x))$ leads to

$$
\begin{aligned}
-1 & +\sum_{x \in G F(p)} \phi(1-x) \phi\left(1-\frac{x}{t}\right)(1+\phi(x)) \\
& =-1+\sum_{x \in G F(p)} \phi(1-x) \phi\left(1-\frac{x}{t}\right)+\sum_{x \in G F(p)} \phi(1-x) \phi\left(1-\frac{x}{t}\right) \phi(x) .
\end{aligned}
$$


This now reduces by Lemma 2.1 and (1.7) to be

$$
=\phi(-t) p_{2} F_{1}(t)-1-\phi(t) .
$$

Now consider

$$
\begin{aligned}
\gamma_{++}(t, p)+\gamma_{+-}(t, p)= & \left|\left\{x \in G F(p)^{\times} \mid \phi\left(1-x^{2}\right)=1, \phi\left(1-\frac{x^{2}}{t}\right) \neq 0\right\}\right| \\
= & \left|\left\{x \in G F(p)^{\times} \mid \phi\left(1-x^{2}\right)=1\right\}\right| \\
& -\left|\left\{x \in G F(p)^{\times} \mid \phi\left(1-x^{2}\right)=1, \phi\left(1-\frac{x^{2}}{t}\right)=0\right\}\right| .
\end{aligned}
$$

The first term is known by Lemma 2.1, and the second is $2(1+\phi(t) / 2)(1+\phi(1-t) / 2)$ since it is 2 if $t$ and $1-t$ are both quadratic residues and 0 otherwise. This leads to

$$
\begin{aligned}
\gamma_{++}(t, p)+\gamma_{+-}(t, p) & =\left(\frac{p-2-\phi(-1)}{2}-1\right)-2\left(\frac{1+\phi(t)}{2}\right)\left(\frac{1+\phi(1-t)}{2}\right) \\
& =\frac{1}{2}\left(p-\phi(-1)-\phi(t)-\phi(1-t)-\phi\left(t-t^{2}\right)-5\right) .
\end{aligned}
$$

Similarly we obtain

$$
\gamma_{++}(t, p)+\gamma_{-+}(t, p)=\frac{1}{2}\left(p-\phi(t)-\phi(-t)-2 \phi\left(t^{2}-t\right)-5\right)
$$

Solving (2.6), (2.9), (2.11), and (2.12) for $\gamma_{++}(t, p), \gamma_{+-}(t, p), \gamma_{-+}(t, p)$, and $\gamma_{++}(t, p)$ produces the result.

Theorem 2.3 depends on auxiliary constants determined by the values of $\phi(-1)$, $\phi(t)$, and $\phi(1-t)$. These constants are defined in Tables A.1, A.2, A.3, A.4, A.5, A.6, A.7, and A.8 in the appendix.

THEOREM 2.3. If $p$ is an odd prime, and $2 \leq t \leq p-1$, then

$$
\begin{aligned}
& |E(i, t, p)| \\
& \quad=\frac{p^{2}+p^{2} \cdot \mathscr{F}(i, t, p) \cdot{ }_{3} F_{2}(t)+p \cdot{ }_{-} 2 F_{1}(t) \cdot \mathscr{G}(i, t, p)+p \cdot \mathscr{H}(i, t, p)+\mathscr{L}(i, t, p)}{32} .
\end{aligned}
$$

Proof. Define $\alpha(i, t, p)$ by

$$
\begin{array}{r}
\alpha(i, t, p):=\mid\left\{(x, y) \in G F(p)^{\times} \times G F(p)^{\times} \mid \phi\left(1-x^{2}\right)=(-1)^{\lfloor(i-1) / 4\rfloor},\right. \\
\left.\phi\left(1-y^{2}\right)=(-1)^{\lfloor(i-1) / 2\rfloor}, \phi\left(x^{2}-t y^{2}\right)=(-1)^{i-1}\right\} \mid .
\end{array}
$$

Thus $\alpha(i, t, p)=4|E(i, t, p)|$. The $\alpha(i, t, p)$ will be determined by solving eight 
equations. First,

$$
\begin{aligned}
\alpha(1, t, p)-\alpha(2, t, p)-\alpha(3, t, p)+\alpha(4, t, p) & -\alpha(5, t, p)+\alpha(6, t, p)+\alpha(7, t, p)-\alpha(8, t, p) \\
= & \sum_{x, y \in G F(p)^{\times}} \phi\left(1-x^{2}\right) \phi\left(1-y^{2}\right) \phi\left(x^{2}-t y^{2}\right) \\
= & \sum_{x, y \in G F(p)} \phi\left(\left(1-x^{2}\right)\left(1-y^{2}\right)\left(x^{2}-t y^{2}\right)\right) \\
& -\sum_{x, y \in G F(p), x y=0} \phi\left(\left(1-x^{2}\right)\left(1-y^{2}\right)\left(x^{2}-t y^{2}\right)\right) \\
= & \sum_{x, y \in G F(p)} \phi((1-x)(1-y)(x-t y))(1+\phi(x))(1+\phi(y)) \\
& +1+\phi(-1)+\phi(t)+\phi(-t) .
\end{aligned}
$$

The above sum involves the four simpler sums

$$
\begin{aligned}
A & :=\sum_{x, y \in G F(p)} \phi((1-x)(1-y)(x-t y)), \\
B & :=\sum_{x, y \in G F(p)} \phi((1-x)(1-y)(x-t y) x), \\
C & :=\sum_{x, y \in G F(p)} \phi((1-x)(1-y)(x-t y) y), \\
D & :=\sum_{x, y \in G F(p)} \phi((1-x)(1-y)(x-t y) x y) .
\end{aligned}
$$

By Lemma 2.1, $A$ and $B$ are given by

$$
\begin{aligned}
A= & \sum_{1 / t \neq y \in G F(p)} \phi(1-y) \sum_{x \in G F(p)} \phi((1-x)(x-t y)) \\
& +\phi\left(1-\frac{1}{t}\right) \sum_{x \in G F(p)} \phi((1-x)(x-1)) \\
= & \phi\left(\frac{1}{t}-1\right)+(p-1) \phi\left(\frac{1}{t}-1\right) \\
= & \phi\left(\frac{1}{t}-1\right) p, \\
B= & \sum_{1 / t \neq y \in G F(p)} \phi\left(y-y^{2}\right) \sum_{x \in G F(p)} \phi((1-x)(x-t y)) \\
& +\phi\left(\frac{1}{t}-\frac{1}{t^{2}}\right) \sum_{x \in G F(p)} \phi((1-x)(x-1)) \\
= & \sum_{1 / t \neq y \in G F(p)} \phi\left(y-y^{2}\right)(-\phi(-1))+(p-1) \phi(1-t) \\
= & 1+\phi(1-t)+(p-1) \phi(1-t) \\
= & \phi(1-t) p+1 .
\end{aligned}
$$


Similarly $C$ can easily be shown to be $C=\phi(-t)+\phi(1-t) p$. By (1.8), $D$ is a simple multiple of ${ }_{3} F_{2}(t)$, which combined with the formulae for $A, B$, and $C$ leads to

$$
\begin{aligned}
\alpha(1, t, p)-\alpha(2, t, p)-\alpha(3, t, p)+\alpha(4, t, p)-\alpha(5, t, p) \\
+\alpha(6, t, p)+\alpha(7, t, p)-\alpha(8, t, p) \\
=\phi(-1)_{3} F_{2}(t) p^{2}+\phi(1-t) 2 p \\
+\phi\left(t-t^{2}\right) p+2+2 \phi(-t)+\phi(-1)+\phi(t) .
\end{aligned}
$$

Each $\alpha(i, t, p)$ can be expressed in terms of $\phi(-1), \phi(t), \phi(1-t),{ }_{2} F_{1}(t)$, and ${ }_{3} F_{2}(t)$. Determining these expressions is no more than solving simple systems of equations. For brevity, we only consider the case where $(\phi(-1), \phi(t), \phi(1-t))=(1,-1,-1)$. The solution in this case determines the entries in Table A.5. In the remaining cases, the tables are determined in exactly the same way.

We first derive an equation for $\alpha(1, t, p)+\alpha(3, t, p)$ in the following way:

$$
\begin{aligned}
\alpha(1, t, p)+\alpha(3, t, p) & \\
= & \left|\left\{(x, y) \in G F(p)^{\times} \times G F(p)^{\times} \mid \phi\left(1-x^{2}\right)=1, \phi\left(1-y^{2}\right) \neq 0, \phi\left(x^{2}-t y^{2}\right)=1\right\}\right| \\
= & \left|\left\{(x, y) \in G F(p)^{\times} \times G F(p)^{\times} \mid \phi\left(1-x^{2}\right)=1, \phi\left(x^{2}-t y^{2}\right)=1\right\}\right| \\
& \quad-2\left|\left\{x \in G F(p) \mid \phi\left(1-x^{2}\right)=1, \phi\left(1-\frac{x^{2}}{t}\right)=\phi(-t)=-1\right\}\right| \\
= & \left|\left\{x \in G F(p)^{\times} \mid \phi\left(1-x^{2}\right)=1\right\}\right| \cdot\left|\left\{y \in G F(p)^{\times} \mid \phi\left(1-t y^{2}\right)=1\right\}\right|-2 \gamma_{+-}(t, p) \\
= & \left(\frac{p-5}{2}\right)\left(\frac{p-1}{2}\right)-\frac{p-7+p_{2} F_{1}(t)}{2} .
\end{aligned}
$$

The following equations are determined in a similar way,

$$
\begin{aligned}
& \alpha(1, t, p)+\alpha(2, t, p)=\left(\frac{p-5}{2}\right)^{2}, \\
& \alpha(3, t, p)+\alpha(4, t, p)=\left(\frac{p-5}{2}\right)\left(\frac{p-1}{2}\right), \\
& \alpha(5, t, p)+\alpha(6, t, p)=\left(\frac{p-1}{2}\right)\left(\frac{p-5}{2}\right), \\
& \alpha(7, t, p)+\alpha(8, t, p)=\left(\frac{p-1}{2}\right)^{2}-\frac{p+1-p_{2} F_{1}(t)}{2}, \\
& \alpha(1, t, p)+\alpha(5, t, p)=\left(\frac{p-5}{2}\right)\left(\frac{p-1}{2}\right)-\frac{p-3+p_{2} F_{1}(t)}{2}, \\
& \alpha(3, t, p)+\alpha(7, t, p)=\left(\frac{p-1}{2}\right)^{2}-\frac{p-1-p_{2} F_{1}(t)}{2} .
\end{aligned}
$$

The solution to the system (2.18), (2.19), and (2.20) for $\alpha(i, t, p)$ for $1 \leq i \leq 8$ yields the entries in Table A.5.

As an immediate consequence of (1.11), Theorems 1.3 and 1.4 we obtain the following result. 
COROLlary 2.4. If $p$ is an odd prime, $2 \leq t \leq p-1$, and $\delta: \equiv(4 t-4) / t(\bmod p)$, then

$$
\begin{aligned}
&|E(i, t, p)|= \frac{p^{2}}{32}(\phi(1-t) \mathscr{F}(i, t, p)+1) \\
&+\frac{p}{32}(-\phi(-1) \mathscr{G}(i, t, p)+\mathscr{H}(i, t, p) \\
&\left.\quad-2 \phi(1-t) \mathscr{F}(i, t, p){ }_{3} N_{2}(\delta, p)-\phi(1-t) \mathscr{F}(i, t, p)\right) \\
&+\frac{1}{32}\left(\phi(1-t) \mathscr{F}(i, t, p){ }_{3} N_{2}(\delta, p)^{2}\right. \\
&\left.+\phi(-1) \mathscr{G}(i, t, p){ }_{2} N_{1}(t, p)+\mathscr{F}(i, t, p)\right) .
\end{aligned}
$$

EXAMPLE 2.5. Consider the graph $G(7,4,13)$. By Corollary 2.4 we find that $\delta \equiv 3$ (mod 13), and by (1.9) we are lead to consider the GF(13) points of the curves

$$
{ }_{2} E_{1}(4): y^{2}=x^{3}-5 x^{2}+4 x, \quad{ }_{3} E_{2}(3): y^{2}=x^{3}-9 x^{2}+27 x-243
$$

Both curves have ${ }_{2} N_{1}(4,13)={ }_{3} N_{2}(3,13)=15$ points over GF(13), and so by Corollary 2.4 (using Table A.1 since $\phi(-1)=\phi(4)=\phi(-3)=1$ ) we find that $|E(7,4,13)|=4$. It is easy to verify that this is indeed true, since the edge set is

$$
E(7,4,13)=\{3 \longrightarrow 3,9 \longrightarrow 3,9 \longrightarrow 9,12 \longrightarrow 12\}
$$

If $t \equiv-1(\bmod p)$, then formulae like (1.1) follow from evaluations proved in [4]

$$
\begin{aligned}
& { }_{2} F_{1}(-1)= \begin{cases}0, & \text { if } p \equiv 3(\bmod 4) ; \\
\frac{2 x(-1)(x+y+1) / 2}{p}, & \text { if } p \equiv 1(\bmod 4), x^{2}+y^{2}=p, x \text { odd. }\end{cases} \\
& { }_{3} F_{2}(-1)= \begin{cases}-\frac{\phi(2)}{p}, & \text { if } p \equiv 5,7(\bmod 8) ; \\
\frac{\phi(2)\left(4 x^{2}-p\right)}{p^{2}}, & \text { if } p \equiv 1,3(\bmod 8), x^{2}+2 y^{2}=p .\end{cases}
\end{aligned}
$$

COROLLARY 2.6. Let $p$ be prime, and define integers $u, v, x$, and $y$ by

$$
\begin{aligned}
& p=x^{2}+y^{2}, \quad \text { if } p \equiv 1(\bmod 4) \text { with } x \text { odd }, \\
& p=u^{2}+2 v^{2}, \quad \text { if } p \equiv 1,3(\bmod 8) .
\end{aligned}
$$

If $p \equiv 1(\bmod 8)$, then

$$
|E(i,-1, p)|=\frac{1}{32} \cdot \begin{cases}p^{2}-12 p-12 x(-1)^{(x+y+1) / 2}+4 u^{2}+91, & \text { if } i=1 ; \\ p^{2}-12 p+4 x(-1)^{(x+y+1) / 2}-4 u^{2}+19, & \text { if } i=1,3 \text { or } 5 \\ p^{2}-4 p+4 x(-1)^{(x+y+1) / 2}+4 u^{2}+3, & \text { if } i=4,6 \text { or } 7 \\ p^{2}-4 p-12 x(-1)^{(x+y+1) / 2}-4 u^{2}-5, & \text { if } i=8 .\end{cases}
$$


If $p \equiv 3(\bmod 8)$, then

$$
|E(i,-1, p)|=\frac{1}{32} \cdot \begin{cases}p^{2}-10 p+4 u^{2}+17, & \text { if } i=1 \text { or } 7 \\ p^{2}-2 p-4 u^{2}+1, & \text { if } i=2 \text { or } 8 \\ p^{2}-6 p-4 u^{2}+13, & \text { if } i=3 \text { or } 5 \\ p^{2}-6 p+4 u^{2}+5, & \text { if } i=4 \text { or } 6 .\end{cases}
$$

If $p \equiv 5(\bmod 8)$, then

$$
|E(i,-1, p)|=\frac{1}{32} \cdot \begin{cases}p^{2}-16 p-12 x(-1)^{(x+y+1) / 2}+67, & \text { if } i=1 \\ p^{2}-8 p+4 x(-1)^{(x+y+1) / 2}+11, & \text { if } 2 \leq i \leq 7 \\ p^{2}-12 x(-1)^{(x+y+1) / 2}+19, & \text { if } i=8 .\end{cases}
$$

If $p \equiv 7(\bmod 8)$, then

$$
|E(i,-1, p)|=\frac{1}{32} \cdot \begin{cases}p^{2}-6 p+25, & \text { if } i=1 \text { or } 7 \\ p^{2}-6 p-7, & \text { if } i=2 \text { or } 8 \\ p^{2}-10 p+21, & \text { if } i=3 \text { or } 5 \\ p^{2}-2 p-3, & \text { if } i=4 \text { or } 6\end{cases}
$$

Even though the only $t$ for which ${ }_{2} F_{1}(t)$ and ${ }_{3} F_{2}(t)$ are known to simultaneously have explicit evaluations are $t=0$ and \pm 1 , we can still obtain simple formulae using the fact that $\mathscr{G}(i, t, p)$ is often zero in Tables A.3, A.5, A.7, and A.8. If $p>3$ is prime, then the following formulae were obtained in [4]:

$$
\begin{aligned}
& { }_{3} F_{2}(-8)= \begin{cases}-\frac{1}{p}, & \text { if } p \equiv 3(\bmod 4) ; \\
\frac{4 x^{2}-p}{p^{2}}, & \text { if } p \equiv 1(\bmod 4), x^{2}+y^{2}=p, x \text { odd } .\end{cases} \\
& { }_{3} F_{2}\left(\frac{-1}{8}\right)= \begin{cases}-\frac{\phi(2)}{p}, & \text { if } p \equiv 3(\bmod 4) ; \\
\frac{\phi(2)\left(4 x^{2}-P\right)}{p^{2}}, & \text { if } p \equiv 1(\bmod 4), x^{2}+y^{2}=p, x \text { odd. }\end{cases}
\end{aligned}
$$

Now as an immediate consequence we obtain the following result.

COROLlary 2.7. Suppose that $p \equiv 5$ or $7(\bmod 8)$ is prime, and that $t \equiv-8$ or $-1 / 8$ $(\bmod p)$. If $p \equiv 5(\bmod 8)$ and $t \equiv-8(\bmod p)$, then

$$
|E(i, t, p)|=\frac{1}{32} \cdot \begin{cases}p^{2}-6 p-4 x^{2}+9, & \text { if } i=3 \text { or } 5 \\ p^{2}-6 p+4 x^{2}+1, & \text { if } i=4 \text { or } 6 .\end{cases}
$$

If $p \equiv 5(\bmod 8)$ and $t \equiv-1 / 8(\bmod p)$, then

$$
|E(i, t, p)|=\frac{1}{32} \cdot \begin{cases}p^{2}-6 p+4 x^{2}+9, & \text { if } i=3 \text { or } 5 \\ p^{2}-6 p-4 x^{2}+1, & \text { if } i=4 \text { or } 6 .\end{cases}
$$


If $p \equiv 7(\bmod 8)$ and $t \equiv-8$ or $-1 / 8$, then

$$
|E(i, t, p)|=\frac{1}{32} \cdot \begin{cases}p^{2}-6 p+25, & \text { if } i=1 \text { or } 7 \\ p^{2}-6 p-7, & \text { if } i=2 \text { or } 8\end{cases}
$$

EXAMPLE 2.8. Consider the graph $G(8,-1,17)$. Since $17=1^{2}+4^{2}=3^{2}+2 \cdot 2^{2}$, Corollary 2.6 with $x=1, y=4, u=3$, and $v=2$ also shows $|E(8,-1,17)|=6$. It is easy to check that the 6 edges are

$$
E(8,-1,17)=\{4 \longrightarrow 8,8 \rightarrow 4,15 \longrightarrow 13,15 \longrightarrow 8,13 \longrightarrow 15,8 \longrightarrow 15\} .
$$

EXAMPLE 2.9. Consider the graph $G(2,15,23)$. Since $23 \equiv 7(\bmod 8)$ and $15 \equiv-8$ $(\bmod 23)$, Corollary 2.7 implies that $|E(2,-8,23)|=12$. It is easy to check that these 12 edges are

$$
\begin{aligned}
& E(2,15,23)=\{16 \longrightarrow 8,16 \longrightarrow 12,18 \longrightarrow 6,18 \longrightarrow 12,16 \longrightarrow 18,12 \longrightarrow 6, \\
& 12 \longrightarrow 8,8 \longrightarrow 6,8 \longrightarrow 18,8 \longrightarrow 16,6 \longrightarrow 12,6 \longrightarrow 16\} \text {. }
\end{aligned}
$$

Now we state the result when $t=1$. Since the proof in this case follows the same type of argument leading to Theorem 2.3, we omit it for brevity.

THEOREM 2.10. If $p$ is an odd prime, then the number of edges of $G(i, 1, p)$ is

$$
|E(i, 1, p)|=\frac{1}{32} \cdot \begin{cases}p^{2}+p^{2} \phi(-1)_{3} F_{2}(1)-15 p-\phi(-1) 5 p+\phi(-1) 30+51, & \text { if } i=1 ; \\ p^{2}-p^{2} \phi(-1){ }_{3} F_{2}(1)-9 p+\phi(-1) p-\phi(-1) 6+15, & \text { if } i=2,5 \text { or } 8 ; \\ p^{2}-p^{2} \phi(-1)_{3} F_{2}(1)-5 p-\phi(-1) 3 p+\phi(-1) 6+3, & \text { if } i=3 ; \\ p^{2}+p^{2} \phi(-1){ }_{3} F_{2}(1)-7 p+\phi(-1) 3 p-\phi(-1) 10+11, & \text { if } i=4 \text { or } 7 ; \\ p^{2}+p^{2} \phi(-1){ }_{3} F_{2}(1)-3 p-\phi(-1) p+\phi(-1) 2-1, & \text { if } i=6 .\end{cases}
$$

Since Evans (see [1]) proved that ${ }_{3} F_{2}(1)=\left(4 x^{2}-2 p\right) p^{2}$ if $p=x^{2}+y^{2}$ where $x$ is odd, and is zero otherwise, we obtain the following corollary.

COROLLARY 2.11. If $p=x^{2}+y^{2}$ is prime where $x$ is odd, then

$$
|E(i, 1, p)|=\frac{1}{32} \cdot \begin{cases}p^{2}-22 p+4 x^{2}+81, & \text { if } i=1 ; \\ p^{2}-6 p-4 x^{2}+9, & \text { if } i=2,3,5, \text { or } 8 \\ p^{2}-6 p+4 x^{2}+1, & \text { if } i=4,6, \text { or } 7 .\end{cases}
$$

If $p \equiv 3(\bmod 4)$ is prime, then

$$
|E(i, 1, p)|=\frac{1}{32} \cdot \begin{cases}p^{2}-10 p+21, & \text { if } i=1,2,4,5,7, \text { or } 8 \\ p^{2}-2 p-3, & \text { if } i=3 \text { or } 6\end{cases}
$$

Since $E(1,1, p)$ is a double cover of $G^{*}(p)$ when $p \equiv 1(\bmod 4)$, the formula for $|E(1,1, p)|$ in the above corollary is equivalent to (1.1). 


\section{Appendix}

TABLE A.1. $(\phi(-1), \phi(t), \phi(1-t))=(1,1,1)$.

\begin{tabular}{rrrcc}
\hline$i$ & $\mathscr{F}(i, t, p)$ & $\mathscr{G}(i, t, p)$ & $\mathscr{H}(i, t, p)$ & $\mathscr{F}(i, t, p)$ \\
\hline 1 & 1 & -6 & -11 & 91 \\
2 & -1 & 2 & -13 & 19 \\
3 & -1 & 2 & -13 & 19 \\
4 & 1 & 2 & -3 & 3 \\
5 & -1 & 2 & -13 & 19 \\
6 & 1 & 2 & -3 & 3 \\
7 & 1 & 2 & -3 & 3 \\
8 & -1 & -6 & -5 & -5 \\
\hline
\end{tabular}

TABLE A.2. $(\phi(-1), \phi(t), \phi(1-t))=(-1,1,1)$.

\begin{tabular}{rrrcc}
\hline$i$ & $\mathscr{F}(i, t, p)$ & $\mathscr{G}(i, t, p)$ & $\mathscr{H}(i, t, p)$ & $\mathscr{J}(i, t, p)$ \\
\hline 1 & -1 & 2 & -5 & 27 \\
2 & 1 & 2 & -11 & 19 \\
3 & 1 & -6 & -11 & 19 \\
4 & -1 & 2 & -5 & 27 \\
5 & 1 & 2 & -11 & 19 \\
6 & -1 & -6 & -5 & -5 \\
7 & -1 & 2 & -5 & 27 \\
8 & 1 & 2 & -11 & 19 \\
\hline
\end{tabular}

TABLE A.3. $(\phi(-1), \phi(t), \phi(1-t))=(1,-1,1)$.

\begin{tabular}{ccccc}
\hline$i$ & $\mathscr{F}(i, t, p)$ & $\mathscr{G}(i, t, p)$ & $\mathscr{H}(i, t, p)$ & $\mathscr{g}(i, t, p)$ \\
\hline 1 & 1 & -4 & -9 & 29 \\
2 & -1 & 4 & -11 & 21 \\
3 & -1 & 0 & -7 & 9 \\
4 & 1 & 0 & -5 & 1 \\
5 & -1 & 0 & -7 & 9 \\
6 & 1 & 0 & -5 & 1 \\
7 & 1 & 4 & -1 & 5 \\
8 & -1 & -4 & -3 & -3 \\
\hline
\end{tabular}


TABLE A.4. $(\phi(-1), \phi(t), \phi(1-t))=(1,1,-1)$.

\begin{tabular}{rrrcc}
\hline$i$ & $\mathscr{F}(i, t, p)$ & $\mathscr{G}(i, t, p)$ & $\mathcal{H}(i, t, p)$ & $\mathscr{J}(i, t, p)$ \\
\hline 1 & 1 & -6 & -17 & 67 \\
2 & -1 & 2 & -7 & 11 \\
3 & -1 & 2 & -7 & 11 \\
4 & 1 & 2 & -9 & 11 \\
5 & -1 & 2 & -7 & 11 \\
6 & 1 & 2 & -9 & 11 \\
7 & 1 & 2 & -9 & 11 \\
8 & -1 & -6 & 1 & 19 \\
\hline
\end{tabular}

TABLE A.5. $(\phi(-1), \phi(t), \phi(1-t))=(1,-1,-1)$.

\begin{tabular}{rrrcc}
\hline$i$ & $\mathscr{F}(i, t, p)$ & $\mathscr{G}(i, t, p)$ & $\mathscr{H}(i, t, p)$ & $\mathscr{F}(i, t, p)$ \\
\hline 1 & 1 & -4 & -11 & 21 \\
2 & -1 & 4 & -9 & 29 \\
3 & -1 & 0 & -5 & 1 \\
4 & 1 & 0 & -7 & 9 \\
5 & -1 & 0 & -5 & 1 \\
6 & 1 & 0 & -7 & 9 \\
7 & 1 & 4 & -3 & -3 \\
8 & -1 & -4 & -1 & 5 \\
\hline
\end{tabular}

TABLE A.6. $(\phi(-1), \phi(t), \phi(1-t))=(-1,1,-1)$.

\begin{tabular}{rrccc}
\hline$i$ & $\mathscr{F}(i, t, p)$ & $\mathscr{G}(i, t, p)$ & $\mathscr{H}(i, t, p)$ & $\mathscr{J}(i, t, p)$ \\
\hline 1 & -1 & 2 & -11 & 19 \\
2 & 1 & 2 & -5 & 27 \\
3 & 1 & -6 & -5 & -5 \\
4 & -1 & 2 & -11 & 19 \\
5 & 1 & 2 & -5 & 27 \\
6 & -1 & -6 & -11 & 19 \\
7 & -1 & 2 & -11 & 19 \\
8 & 1 & 2 & -5 & 27 \\
\hline
\end{tabular}


TABLE A.7. $(\phi(-1), \phi(t), \phi(1-t))=(-1,-1,1)$.

\begin{tabular}{ccccc}
\hline$i$ & $\mathscr{F}(i, t, p)$ & $\mathscr{G}(i, t, p)$ & $\mathscr{H}(i, t, p)$ & $\mathscr{F}(i, t, p)$ \\
\hline 1 & -1 & 0 & -7 & 25 \\
2 & 1 & 0 & -5 & -7 \\
3 & 1 & -4 & -9 & 21 \\
4 & -1 & 4 & -3 & -3 \\
5 & 1 & 4 & -9 & 21 \\
6 & -1 & -4 & -3 & -3 \\
7 & -1 & 0 & -7 & 25 \\
8 & 1 & 0 & -5 & -7 \\
\hline
\end{tabular}

TABLE A.8. $(\phi(-1), \phi(t), \phi(1-t))=(-1,-1,-1)$.

\begin{tabular}{rrrcc}
\hline$i$ & $\mathscr{F}(i, t, p)$ & $\mathscr{G}(i, t, p)$ & $\mathscr{H}(i, t, p)$ & $\mathscr{f}(i, t, p)$ \\
\hline 1 & -1 & 0 & -9 & 17 \\
2 & 1 & 0 & -3 & 1 \\
3 & 1 & -4 & -7 & 13 \\
4 & -1 & 4 & -5 & 5 \\
5 & 1 & 4 & -7 & 13 \\
6 & -1 & -4 & -5 & 5 \\
7 & -1 & 0 & -9 & 17 \\
8 & 1 & 0 & -3 & 1 \\
\hline
\end{tabular}

ACKNOWLEDGement. The author wishes to express thanks to Ken Ono for suggesting this investigation and for numerous helpful suggestions during the preparation of this article.

\section{REFERENCES}

[1] R. J. Evans, Identities for products of Gauss sums over finite fields, Enseign. Math. (2) 27 (1981), no. 3-4, 197-209 (1982). MR 83i:10050. Zbl 491.12020.

[2] R. J. Evans, J. R. Pulham, and J. Sheehan, On the number of complete subgraphs contained in certain graphs, J. Combin. Theory Ser. B 30 (1981), no. 3, 364-371. MR 83c:05075. Zbl 475.05049 .

[3] J. Greene, Hypergeometric functions over finite fields, Trans. Amer. Math. Soc. 301 (1987), no. 1, 77-101. MR 88e:11122. Zbl 629.12017.

[4] K. Ono, Values of Gaussian hypergeometric series, Trans. Amer. Math. Soc. 350 (1998), no. 3, 1205-1223. MR 98e:11141. Zbl 910.11054.

Lawrence Sze: Department of Mathematics, California Polytechnic University, SAN LUIS OBISPO, CA 93407, USA

E-mail address: 1sze@ca1poly.edu 


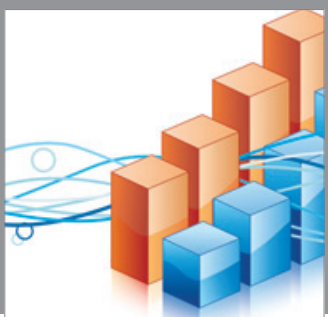

Advances in

Operations Research

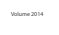

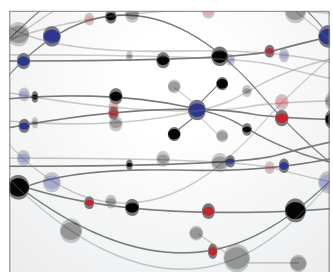

\section{The Scientific} World Journal
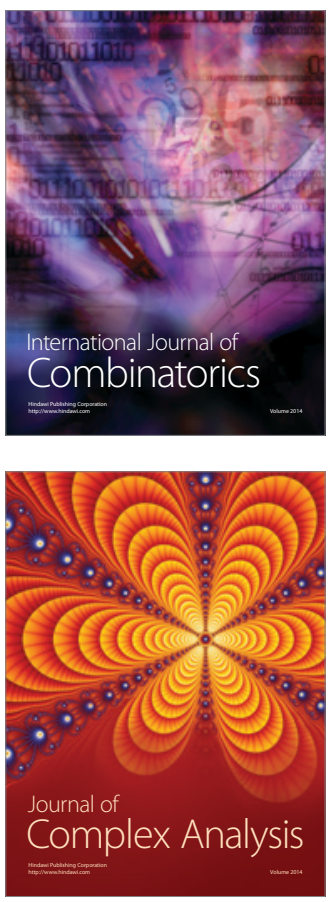

International Journal of

Mathematics and

Mathematical

Sciences
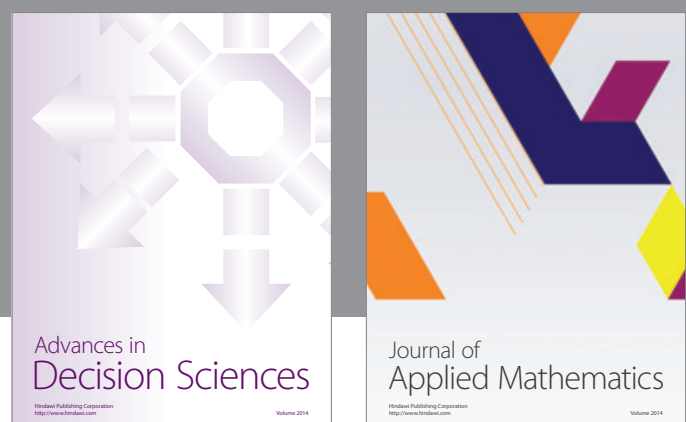

Journal of

Applied Mathematics
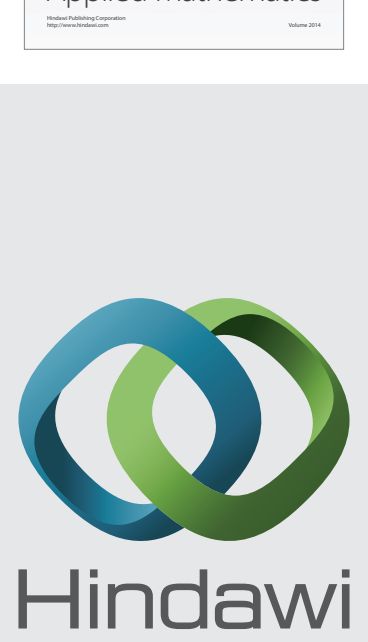

Submit your manuscripts at http://www.hindawi.com
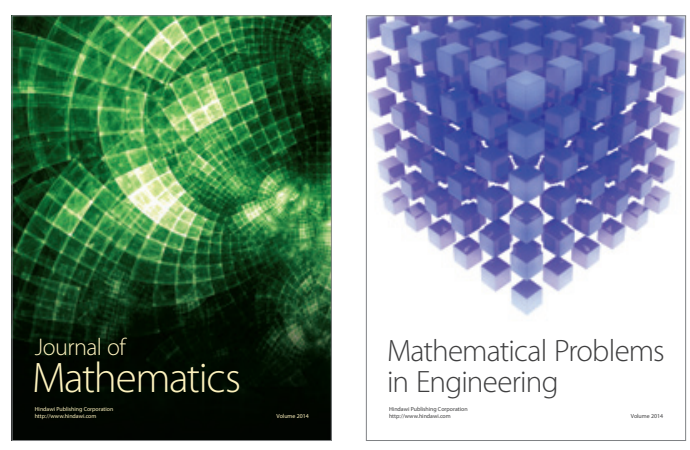

Mathematical Problems in Engineering
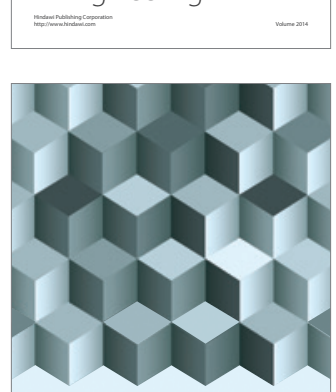

Journal of

Function Spaces
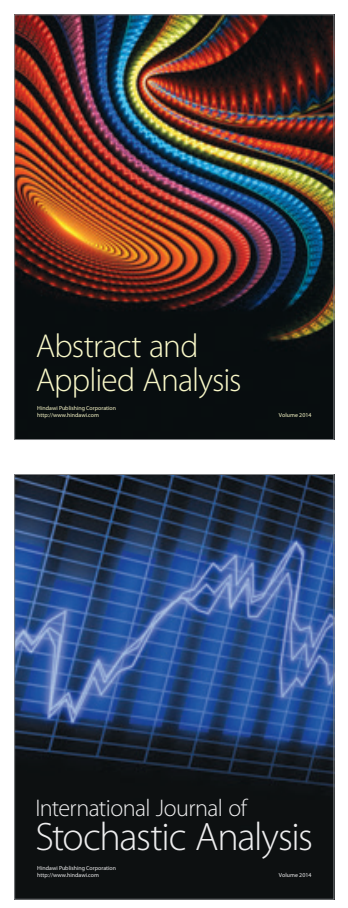

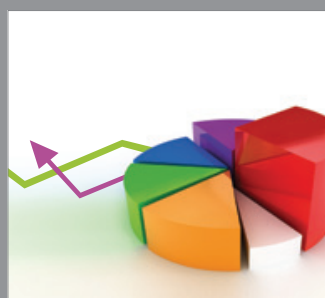

ournal of

Probability and Statistics

Promensencen
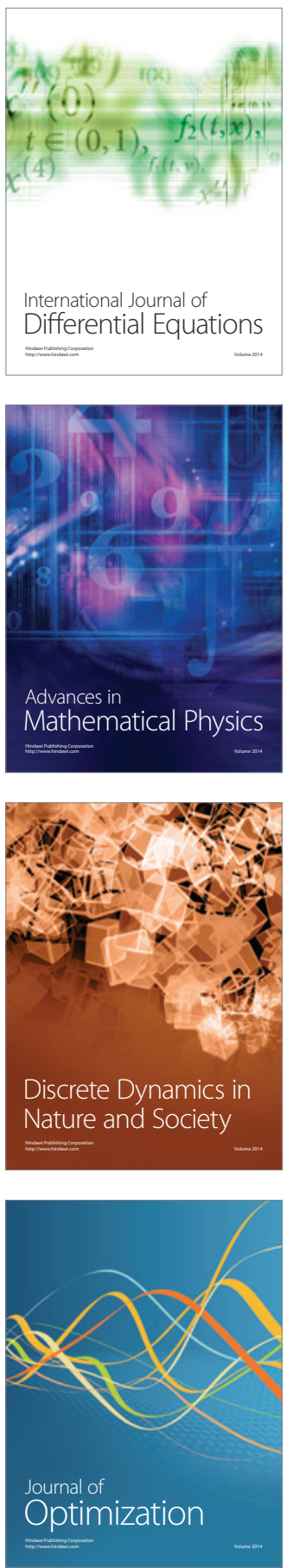